\section{Surface-adhesive and osteogenic self-assembled peptide nanofibers for bioinspired functionalization of titanium surfaces $\dagger$}

\author{
Hakan Ceylan, $\$$ Samet Kocabey, $\$$ Ayse B. Tekinay* and Mustafa O. Guler* \\ Received 17th January 2012, Accepted 3rd February 2012 \\ DOI: $10.1039 / \mathrm{c} 2 \mathrm{sm} 25127 \mathrm{~b}$
}

\begin{abstract}
Mechanical properties and biological inertness of titanium provide potential in orthopedic and dental implants. However, integration of titanium-based implants into the existing tissue is a major problem. Herein, we demonstrate biofunctionalization of titanium surfaces through a mussel-inspired adhesion mechanism conjugated to self-assembled peptide nanofibers in order to overcome biocompatibility issues. A Dopa conjugated peptide nanofiber coating was used along with bioactive peptide sequences for osteogenic activity to enhance osseointegration of medical grade titanium surface, TiA16V4 alloy. Dopa-mediated immobilization of osteogenic peptide nanofibers on titanium surfaces created an osteoconductive interface between osteoblast-like cells and inhibited adhesion and viability of soft tissue forming fibroblasts compared to the uncoated titanium substrate. This biofunctionalization strategy can be extended into other surface immobilization systems owing to the versatile adhesive properties of Dopa and the ease of ligand conjugation to peptide amphiphile molecules.
\end{abstract}

\section{Introduction}

Titanium-based materials have been widely used as orthopedic and dental implants because of their mechanical properties and biological inertness. ${ }^{1,2}$ A major concern with titanium implants is integration into the existing tissue. When bone cells cannot adhere to the surface of the implanted material, the implant cannot be integrated and will eventually detach from the body in the long term. Guiding cellular behaviors (e.g. adhesion, morphogenesis, viability, proliferation, migration and differentiation) has been a critical concern for enhancing osseointegration. In order to overcome tissue integration problems, most of the past and current research has concentrated on modification of bone implants' surface properties by increasing roughness and altering surface chemistry, mostly coating the surface with an oxide layer or immobilizing hydroxyapatite..$^{2-7}$ On the other hand, modification of implant surfaces with biologically active species (e.g. triggering cellular signaling and cues to mimic extracellular matrix) has recently emerged as a promising approach to enhance osseointegration. ${ }^{3,8-12}$ The extracellular matrix (ECM) constituents regulate cellular behaviors in natural

Institute of Materials Science and Nanotechnology, National Nanotechnology Research Center (UNAM), Bilkent University, Ankara, Turkey,06800.E-mail: moguler@unam.bilkent.edu.tr; atekinay@unam. bilkent.edu.tr

$\dagger$ Electronic supplementary information (ESI) available: A detailed description of experimental set-ups, LC-MS characterization and the design principles of peptide amphiphiles, characterization of the nanofiber formation, additional surface characterizations, and figures relating to in vitro experiments. See DOI: $10.1039 / \mathrm{c} 2 \mathrm{sm} 25127 \mathrm{~b}$

$\$$ Equal contribution to the present work. cellular microenvironment by providing cells with spatially and temporally controlled bioactive signals.

The ability of self-assembled peptide amphiphile nanofibers to mimic ECM has attracted intensive interest in recent years. Such biodegradable nanoscale matrices created by these nanofibers have similar structural and mechanical properties to the native ECM. The flexibility in their molecular design allows conjugation of a wide range of bioactive sequences, which are efficiently presented to the cells to promote cellular adhesion, proliferation and differentiation both in in vitro and in vivo environments. ${ }^{10,11,13-15}$ These characteristics are solely controlled through the design of the building blocks that form the nanofibers. ${ }^{3,10,15-17}$ We recently reported a novel biointerface that mediates endothelial cell-selective adhesion and survival on a stainless steel surface. The peptide nanofibers were used to mimic the adhesion strategy of mussels and were conveniently immobilized onto the stainless steel surface along with a fibronectin-derived endothelial cell-specific adhesion epitope, REDV. ${ }^{17}$ A similar approach can be exploited to promote osteogenic activity on titanium implants, since promoting osteoblast adhesion and survival on titanium in a selective manner is a challenging task. Rapid and selective adhesion and growth of osteoblasts on implant surfaces are critical because delayed healing can cause fibroblast-mediated scar tissue formation leading to tissue softening around the implant and requirement for further revision surgeries. ${ }^{18-21}$ The KRSR peptide epitope found in the heparin binding protein in the ECM is known to bind to transmembrane proteoglycans and observed to promote selective adhesion of osteoblasts, while inhibiting the adhesion of fibroblasts. ${ }^{22-24}$ 
Although novel soft bioactive interface materials enhancing biocompatibility and durability of the implant is an emerging choice, most of the available materials suffer from insufficient stability in the aqueous environment. Functionalization of the implant surface such as biotin-streptavidin and nitriloacetic acid-histidine interactions, provide a reversible adhesion platform under controlled conditions, however these techniques are weak in terms of adhesion strength and require surface preparation prior to immobilization. Covalent attachment techniques including $N$-hydroxysuccinimide (NHS)-ethyl (dimethylaminopropyl) carbodiimide (EDC) coupling, ensure strong surface binding and thus offer a wider range of applications. Nevertheless, in addition to the persisting need for surface preparation, these systems are generally susceptible to hydrolysis, that lowers the efficiency of immobilization, and their degradation products may cause biocompatibility issues. ${ }^{25-27}$

To overcome disadvantages of the currently available adhesive methods for medical applications, a sessile organism, mussel, offers a valuable strategy that allows adhesion to inorganic and organic surfaces in the presence of water. Mussels adhere to surfaces via special adhesive proteins that are highly enriched with 3,4-dihydroxy-L-phenylalanine (Dopa), which forms strong bonds with hydrophilic surfaces and complexes with metal ions and metal oxides. ${ }^{28}$ Thus, conjugating Dopa to synthetic materials has attracted growing attention not only because it can operate under aqueous conditions without requiring any surface preparation but also because it is fully biocompatible..$^{25,28-30} \mathrm{In}$ terms of adhesion strength, Dopa adhesion displays a covalent character whilst it is resistant to hydrolysis and is fully reversible. ${ }^{25,31}$ Lee et al. measured the dissociation force between Dopa and $\mathrm{TiO}_{2}$ as $805 \mathrm{pN}$, which is beginning to approach the dissociation force of a typical carbon-silicon covalent bond $(2000 \mathrm{pN})$ and is much higher than the dissociation force of hydrogen bonds that hold the DNA double helix intact $(10-20 \mathrm{pN})$, indicating the strength of the adhesion formed between Dopa and $\mathrm{TiO}_{2}{ }^{31,32}$

In this work, we investigated one-step immobilization of bone ECM-mimetic self-assembled peptide nanofibers on titanium surfaces exploiting mussel adhesion chemistry (Fig. 1B). For this purpose, we used medical grade titanium alloy, TiAl6V4, which is denoted here simply as titanium substrate for clarity. We designed and synthesized a peptide amphiphile (PA) molecule covalently conjugated to Dopa (Lauryl-VVAGE-Dopa$\mathrm{Am})$ for titanium surface functionalization and another PA molecule conjugated to a heparin-binding adhesion peptide sequence, KRSR (Lauryl-VVAGKRSR-Am) to promote osteogenic activity (Fig. 1A and S1A, B, ESI†े). These molecules self-assemble to form a nanofibrous network at physiological $\mathrm{pH}$. In order to investigate the benefit of Dopa residues on the PA molecule, a PA molecule (E-PA) without the Dopa residue (Lauryl-VVAGE) (Fig. 1A and S1C, ESI†े) was also synthesized. The utility of the KRSR peptide in the PA construct was also tested by using a PA molecule (K-PA) that lacked the KRSR sequence but retained the rest of the peptide sequence (Lauryl-VVAGK-Am) (Fig. 1A and S1D, ESI $\dagger$ ). We exploited mussel adhesion chemistry along with osteogenic signals on titanium surfaces in order to selectively promote adhesion, viability and osteogenic activity of osteoblast-like Saos2 cells and to inhibit adhesion and growth of osteolytic gingival fibroblasts. ${ }^{33}$

\section{Results and discussion}

The KRSR peptide functionalized PA (KRSR-PA) and Dopa conjugated PA (Dopa-PA) molecules were synthesized by using a solid-phase peptide synthesis method. Electrostatic stabilization triggered self-assembly of oppositely charged KRSR-PA and Dopa-PA molecules at $\mathrm{pH} 7.4$ into growing nanofibers that elongate via $\beta$-sheets formed in the direction of elongation. ${ }^{34} \mathrm{We}$ visualized the nanofibrous and porous network formed following the self-assembly of KRSR-PA and Dopa-PA using scanning electron microscopy (SEM) (Fig. 2A) and scanning transmission electron microscopy (STEM) (Fig. 2B). We performed circular dichroism analysis (Fig. 2E) to characterize KRSR-PA/DopaPA nanofibers and homogenous distribution of the building blocks within the nanofibers. Neither Dopa-PA nor KRSR-PA formed an organized structure by themselves in solution at $\mathrm{pH}$ 7.4. However, upon mixing, they predominantly formed $\beta$-sheet structures within seconds, indicating $\beta$-sheet-driven nanofiber formation. Rheology measurements of the KRSR-PA and Dopa-PA mixture further confirmed formation of a gel at $\mathrm{pH} 7.4$ that is stabilized by the physical entanglements of nanofibers, a reminiscent feature of native ECM (Fig. $2 \mathrm{C}$ and S2B, ESI†). The self-assembly process was further verified by using zeta potential measurements, as mixing two oppositely charged PA molecules brought the charge of the system up to zero at $\mathrm{pH} 7.4$ (Fig. 2D and S2A, ESI $\dagger$ ).

Osteoconductive modification of the titanium surface is crucial when long-term contact between the implant surface and surrounding cells is required. For this purpose, the binding of the KRSR-PA/Dopa-PA nanofibers on the titanium surface was investigated in the presence of water using X-ray photoelectron spectroscopy (XPS). The coated titanium surfaces were analyzed with XPS after washing. The complete suppression of a photoelectron signal from the titanium substrate and the emergence of a strong nitrogen signal along with increased carbon signal after washing were considered as evidence for the permanent adsorption of KRSR-PA/Dopa-PA nanofibers and the formation of a peptide surface coating (Fig. 3A). To study the role of Dopa in the surface adhesion mechanism, we tested KRSR-PA/ E-PA nanofibers under identical conditions. These nanofibers were readily washed away from the surface in the rinsing steps and hence did not form a peptide layer (Fig. 3A) as shown by a dramatically lowered nitrogen photoelectron peak. Therefore, we concluded that Dopa incorporation is critical for immobilization of peptide nanofibers on the titanium surface. We further showed that KRSR-PA/Dopa-PA nanofibers can adhere onto stainless steel and silicon wafer surfaces, providing insight into the versatility of this system on different surfaces (Fig. S6, ESI $\dagger$ ). An SEM image of the KRSR-PA/Dopa-PA coated titanium surface after washing also revealed that the adsorbent species on the coated titanium surface were indeed peptide nanofibers (Fig. 3B). To support this argument, we characterized KRSRPA/Dopa-PA nanofibers adsorbed on the titanium surface with FT-IR, which revealed characteristic signals similar to previously reported Mefp-1 protein coating adsorbed on $\mathrm{ZnSe}$ surface (Fig. 3C) ${ }^{35}$ Mefp1 mainly consists of Dopa-containing repetitive sequences in its protein structure and constitutes one of the major proteins in the mussel adhesive system. The KRSR-PA/Dopa-PA FT-IR spectrum revealed amide I, amide II and Dopa-specific 


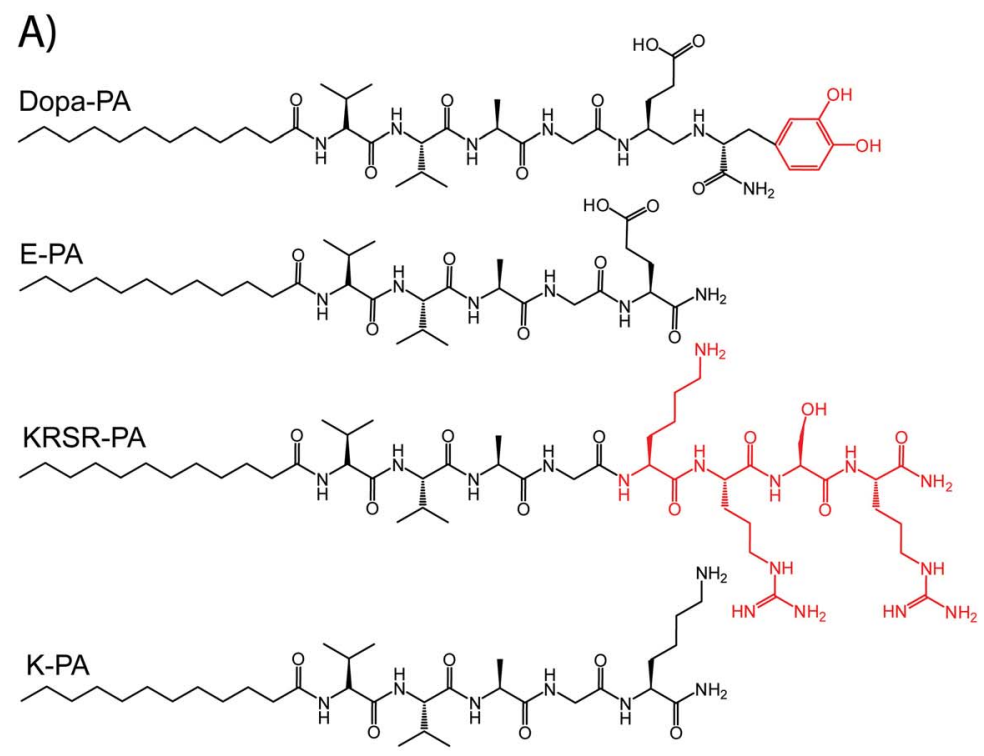

B)

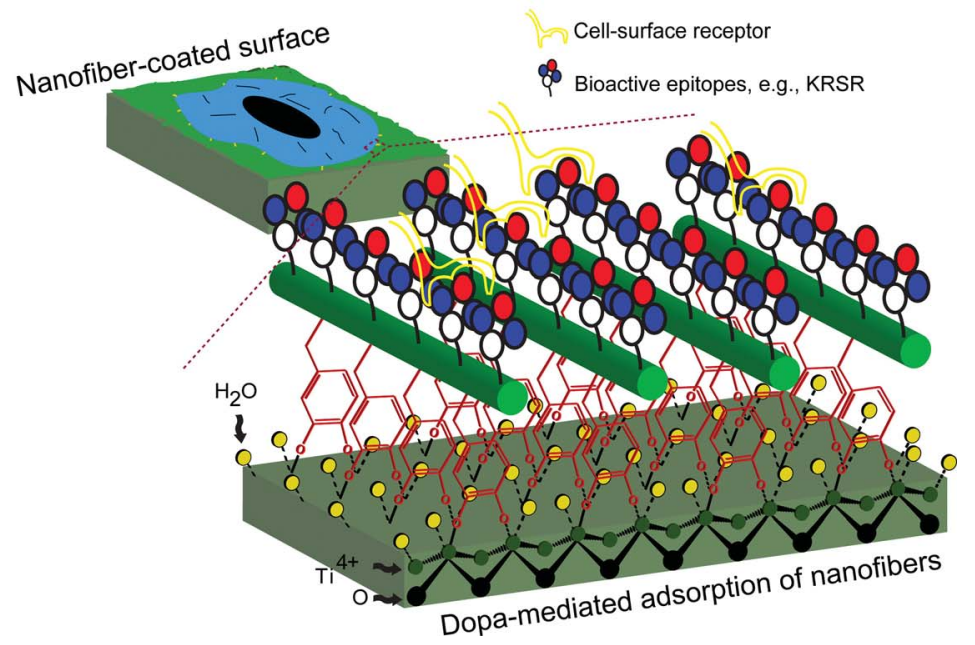

Fig. 1 Schematic illustration of our immobilization strategy for osteogenic nanofibers on titanium surface based on the self-assembly of the KRSR-PA and Dopa-PA. (A) Chemical structures of the peptide amphiphile molecules designed for functionalization of titanium surfaces. (B) Dopa-mediated immobilization of the bioactive nanofibers on titanium surface in the presence of water is shown to occur through catechol-titanium coordination ${ }^{43}$

peaks (see ESI† for all peak assignments and further discussion). On the other hand, FT-IR analysis of the KRSR-PA/E-PA treated surface did not exhibit distinctive peptide signals after rinsing.

Investigation of surface properties is critical, since osteoblasts and fibroblasts, as many other cells do, respond to the chemistry, hydrophilicity and topography of the surface which altogether determine the success of the implant. ${ }^{6}$ Since increased hydrophilicity and roughness are known to promote osteogenic activity, techniques such as titanium plasma spraying, oxide layer formation, acid etching and electrochemical anodization have previously been employed to roughen the surface and increase the surface hydrophilicity. ${ }^{36}$ The titanium surfaces with KRSRPA/Dopa-PA nanofibers showed more hydrophilic characteristics compared to bare titanium surface owing to the supramolecular nanostructures. Mainly, the design of PA molecules dictates the hydrophilic ends of the PA molecules exposed to the aqueous environment, thereby significantly contributing to surface hydrophilicity. For this reason, the contact angle value of the modified surface decreased from 55.2 ${ }^{\circ}$ (bare titanium substrate) to below $17^{\circ}$ (Fig. S3A, ESI $\dagger$ ). For the same reason, a similar contact angle $\left(<17^{\circ}\right)$ decrease was observed on K-PA/ Dopa-PA nanofiber modified titanium surface (Fig. S3C, ESI $\dagger$ ). We also investigated the surface topography and the coating homogeneity of KRSR-PA/Dopa-PA modified titanium surface by using optical profilometer (Fig. 3D and S3B, ESI $\dagger$ ). The surface roughness was found to increase on both KRSR-PA/ Dopa-PA and K-PA/Dopa-PA coated titanium surfaces.

We utilized the sensitivity of contact angle measurement technique to assess the retention of the KRSR-PA/Dopa-PA coating. Ultrasound sonication is a powerful technique to break apart non-covalent molecular interactions. After $1 \mathrm{~h}$ sonication treatment, the contact angle of the surface increased from $<17$ to $33.1^{\circ}$, indicating that despite some of the coating becoming 
A)

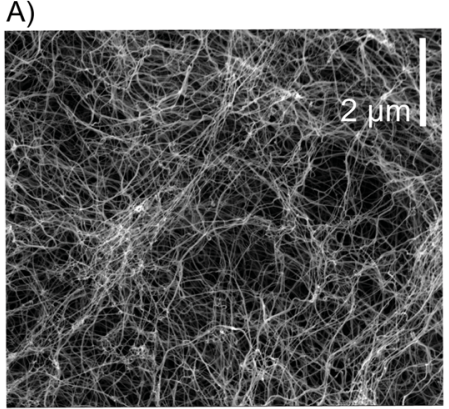

C)

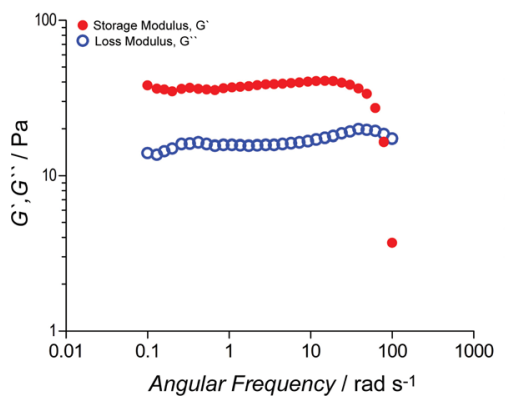

B)

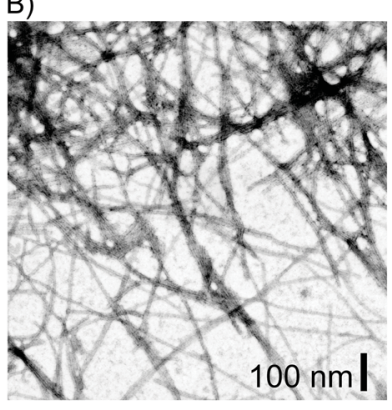

E)
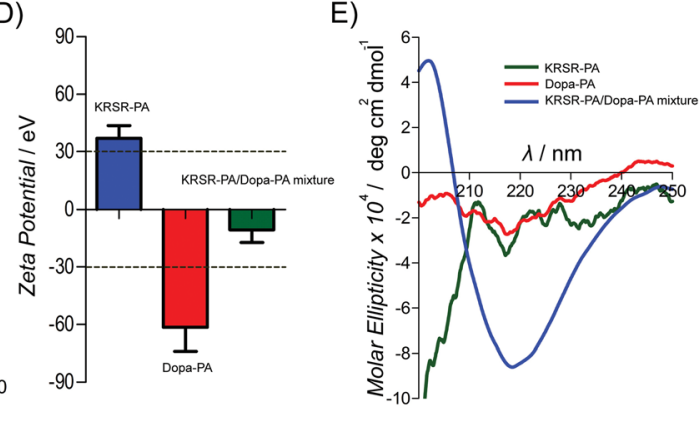

Fig. 2 Characterization of the self-assembled peptide nanofibers and the mechanism of assembly. (A) SEM, (B) STEM images of the KRSR-PA/DopaPA nanofibers formed at $\mathrm{pH}$ 7.4. (C) The mechanical properties of the KRSR-PA/Dopa-PA gel under varying angular frequencies. (D) Zeta potential measurements of KRSR-PA, Dopa-PA and their mixture, KRSR-PA/Dopa-PA. (E) Circular dichroism measurements of KRSR-PA, Dopa-PA and their mixture, KRSR-PA/Dopa-PA.

detached, a significant portion of the nanofibers still remained strongly bound to the surface (Fig. S3A, ESI $\dagger$ ). SEM images after sonication showed similar nanofibrous morphology as shown in Fig. 3B. The relative increase in contact angle can be ascribed to removal of nanofibers that were not bound to the surface through Dopa-mediated titanium-catechol complexes; rather the nanofibers that were removed had originally bound to the surface by physical entanglement of nanofibers. On the other
A)

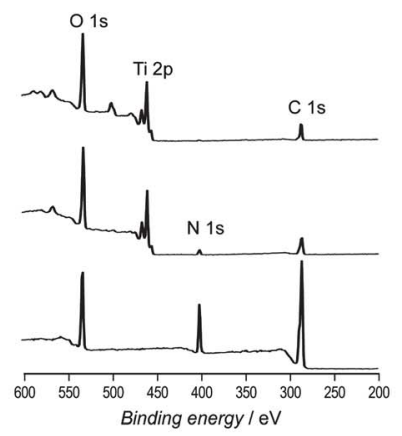

C)

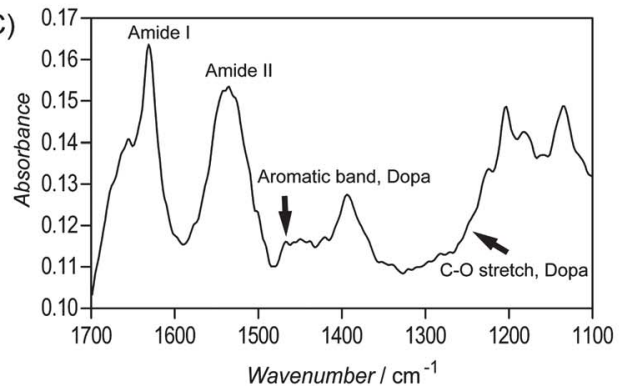

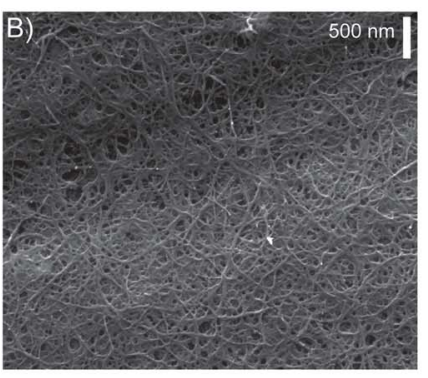

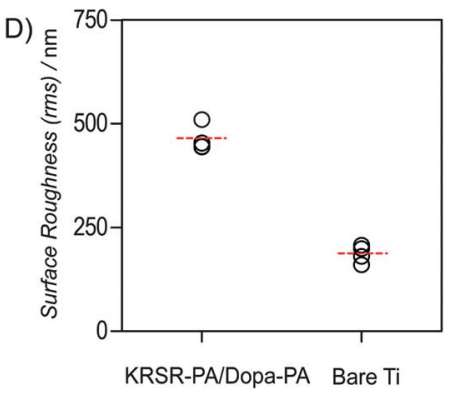

Fig. 3 Specific immobilization of KRSR-PA/Dopa-PA nanofibers on titanium surface. (A) XPS spectra of (top to bottom) bare titanium, KRSR-PA/ E-PA and KRSR-PA/Dopa-PA coated titanium surfaces. (B) SEM micrograph of immobilized KRSR-PA/Dopa-PA nanofibers on titanium surface. (C) ATR/FT-IR spectrum of KRSR-PA/Dopa-PA nanofibers adhered on titanium surface. (D) The roughness of KRSR-PA/Dopa-PA coated and bare titanium surfaces. 
hand, the remaining nanofibers after sonication remained attached to the surface through the near-covalent strength of Dopa binding, which kept the contact angle below $55.2^{\circ}$. The long term stability of the coating was evident with visual observation. The Dopa-PA/KRSR-PA coating remained on the titanium surface in various solvents, including water, acetone, PBS, $10 \%$ Fetal Bovine Serum containing PBS, ethanol and 2-propanol even after more than 2 months.

Cellular behaviors at the bone-implant interface tightly dictate the long term success of the biomaterial and the fate of the regenerating tissue. Healing time, which is a measure of osteoblast adhesion, growth and mineral deposition on the implant, is critical as prolonged healing time causes the risk of tissue softening. For this reason, we first investigated the adhesion and spreading of the osteoblasts on titanium surface coated with KRSR-PA/Dopa-PA nanofibers. Adhesion and spreading of osteoblasts on the implant surface is crucial as an indication of implant bioactivity and the future behavior of cells on this surface. The in vitro results revealed that the number of osteoblastic Saos 2 cells adhered on KRSR-PA/Dopa-PA coated titanium surface at $1 \mathrm{~h}$ was $2.96 \pm 0.19$-fold greater compared to the bare surface (Fig. 4A, unless otherwise specified all \pm s.e.m values are quoted at $p<0.0001$ ) while the number of Saos2 cells adhered on K-PA/Dopa-PA nanofibers was $2.51 \pm 0.08$-fold greater than the bare surface. Clearly the number of adhered Saos 2 cells on KRSR-PA/Dopa-PA was significantly higher than on K-PA/Dopa-PA, highlighting the role of KRSR in mediating osteoblast adhesion. The bioactivity provided by peptide nanofibers was further investigated by analyzing the adhesion behavior of MC3T3-E1 pre-osteoblasts. The results were found to be in parallel with Saos2 adhesion, where cells adhered significantly in greater numbers on KRSR-PA/Dopa-PA compared to both K-PA/Dopa-PA and the bare surface (Fig. S4A, ESI $\dagger$ ). The increased adhesion of the osteoblasts and pre-osteoblasts on KRSR-lacking nanofibers compared to the bare metal surface can be explained by the altered surface properties due to peptide coating. It was previously reported that increased surface roughness and hydrophilicity promoted adhesion of osteoblasts. ${ }^{6,76} \mathrm{We}$ showed that surface roughness and hydrophilicity of K-PA/Dopa-PA coated titanium surface increased to a parallel level of the KRSR-PA/Dopa-PA coated surface, which may significantly contribute to our observation (Fig. S3, ESI + ). In addition, it is known that osteoblast adhesion, proliferation and alkaline phosphatase (ALP) activity are enhanced with nanostructures, and thus could further explain the observation made here. ${ }^{37,38}$ On the other hand, the number of human gingival fibroblasts (HGF) adhered on KRSR-PA/DopaPA coated titanium surface decreased to $0.74 \pm 0.06$-fold compared to K-PA/Dopa-PA and $0.46 \pm 0.03$-fold compared to bare titanium surface. The difference between KRSR-PA/DopaPA and K-PA/Dopa-PA shows the inhibitory role of KRSR toward fibroblast adhesion. ${ }^{22}$ In addition, HGF adhesion on KPA/Dopa-PA coated titanium surface was $0.61 \pm 0.05$-fold compared to the bare titanium surface. No other physical or chemical difference between these two coatings is expected. In addition, we observed that the fibroblast adhesion on K-PA/ Dopa-PA decreased to $0.86 \pm 0.06(p<0.05)$ of the bare surface. This finding is also supported by previous reports, which revealed that gingival fibroblasts favor hydrophobic and
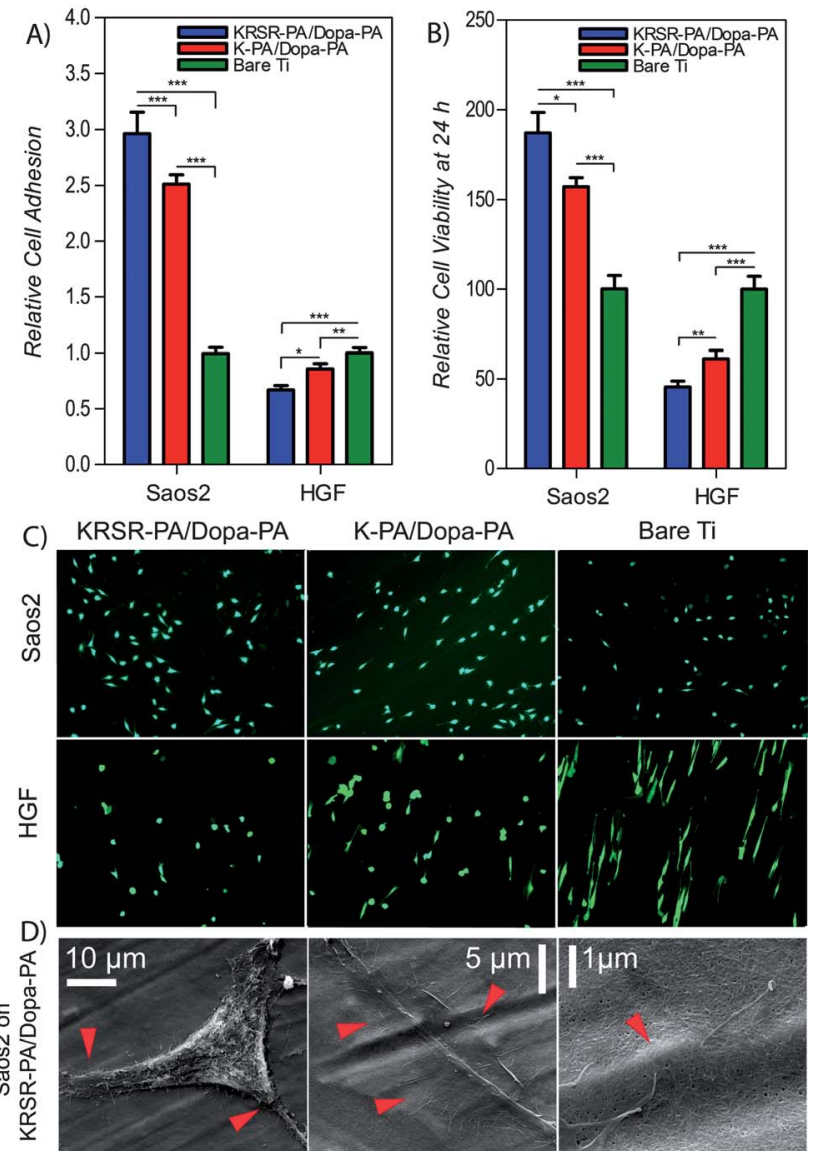

Fig. 4 Adhesion, viability and morphology of cells on functionalized titanium surfaces. Adhesion (A) and viability (B) of Saos 2 and HGF cells on functionalized titanium surfaces. (C) Representative calcein AMstained micrographs $(10 \times$ magnification) of Saos 2 and HGF cells captured on functionalized titanium surfaces at $24 \mathrm{~h}$. (D) Interactions between Saos 2 and KRSR-PA/Dopa-PA coating captured using SEM at $24 \mathrm{~h}$. Red arrows indicate the places at which cells make contact with the matrix; ${ }^{*} p<0.05,{ }^{* *} p<0.01,{ }^{* * *} p<0.0001$.

smoother surfaces, rather than rough and hydrophilic surfaces. ${ }^{39}$ Therefore, our designed PA coatings could selectively favor osteoblast adhesion.

In order to see the effect of the surface properties on cell viability, we incubated osteoblasts and fibroblasts on KRSR-PA/ Dopa-PA coated titanium surfaces for $24 \mathrm{~h}$. We found that Saos2 cell viability gained a selective enhancement on KRSR-PA/ Dopa-PA, whilst fibroblast viability decreased on that coating (Fig. 4B, C and S5, ESI $\dagger$ ). The number of viable Saos2 cells on the KRSR-PA/Dopa-PA coated titanium surface was $1.85 \pm$ 0.19 -fold greater compared to the cells on the bare surface and $1.17 \pm 0.15(p<0.05)$ fold greater than on the K-PA/Dopa-PA coated surface. The viability of Saos2 cells was also favored 1.58 \pm 0.15 -fold on K-PA/Dopa-PA with respect to bare titanium surface at $24 \mathrm{~h}$. These results revealed that even though surface characteristics, such as hydrophilicity and roughness are important factors in Saos2 adhesion and viability, the KRSR epitope also plays significant role as shown by the statistical analysis. A similar observation on the impact of surface hydrophilicity and roughness on cell viability was also reported 
previously. ${ }^{24} \mathrm{We}$ found that the viability of MC3T3-E1 cells was comparable on all tested surfaces (Fig. S4B, ESI $\dagger$ ). In contrast, HGF viability dramatically decreased on PA coated surfaces. The viability of HGF decreased to almost $50 \%$ on KRSR-PA/ Dopa-PA $(0.55 \pm 0.09$-fold $)$ and $75 \%$ on K-PA/Dopa-PA $(0.75 \pm$ 0.12 -fold) compared to bare titanium surface. We noticed that KRSR plays a strong inhibitory role on fibroblast viability. The viability of HGF cells decreased $0.73 \pm 0.12$-fold on KRSR-PA/ Dopa-PA with respect to K-PA/Dopa-PA. These cells attained round-like morphology on rougher and more hydrophilic PA coatings, which indicated an unfavorable microenvironment (Fig. 4C). Actin filament-stained HGFs further showed the loss of their characteristic elongated shapes on both KRSR-PA/ Dopa-PA and K-PA/Dopa-PA coatings at $24 \mathrm{~h}$ (Fig. 4C, S8, ESI $\dagger$ ). On the other hand, Saos2 cells attained their native morphology on PA coated surfaces before they do on bare titanium substrate. Considering cell adhesion, viability and morphology of the cells, the KRSR-PA/Dopa-PA nanofibers provided a favourable microenvironment for osteoblast-like cells (Fig. 4D), while creating an inhibitory microenvironment for fibroblast cells.

As a long term marker for osteoblast adaptivity, we analyzed differentiation and mineral deposition of Saos 2 cells on coated and bare surfaces. ALP activity is an early marker of osteoblastspecific phenotype that is significantly up-regulated during the early phases of osteogenic differentiation. ${ }^{40}$ ALP activity of Saos 2 cells on all surfaces reached its peak value on day 3 and was doubled on KRSR-PA/Dopa-PA and K-PA/Dopa-PA coated surfaces compared to the bare surface (Fig. 5A). On the following days, the ALP activity of Saos 2 cells on coated surfaces remained significantly higher than those on the bare titanium. To test the mineral deposition of Saos-2 cells as a long term response to the titanium surface, Alizarin Red staining was performed on day 14 and 21 and calcium deposition was quantified by extracting Alizarin-Red bound calcium from the surface via cetylpyridinium chloride and measuring its absorbance at 562 $\mathrm{nm}$. The results demonstrated significantly enhanced calcium deposition stained with Alizarin-Red on PA coated titanium surfaces compared to bare titanium surfaces (Fig. 5B) and the amount of Alizarin Red bound calcium was significantly higher on KRSR-PA/Dopa-PA (1.34 \pm 0.1 -fold, $p<0.05)$ and K-PA/ Dopa-PA $(1.29 \pm 0.09$-fold, $p<0.05)$ coatings compared to bare titanium surface (Fig. 5C). Similar mineral deposition pattern was also seen on day 21 for KRSR-PA/Dopa-PA $(2.04 \pm 0.15$ fold) and K-PA/Dopa-PA (1.75 \pm 0.18 -fold) coatings compared to bare titanium surface (Fig. S7, ESI $†$ ). These results showed that the PA coated titanium surfaces exhibit highly enhanced capability of inducing differentiation into osteogenic lineage and mineralization of extracellular matrix. However, the KRSR epitope does not significantly contribute to ALP activity and mineral deposition.

\section{Conclusions}

Dopa-mediated immobilization of osteogenic peptide nanofibers on titanium surfaces created an osteoconductive interface between osteoblast-like cells and the titanium substrate. In addition, the bioactive surface coating inhibited adhesion and viability of soft tissue forming fibroblasts compared to the
A)

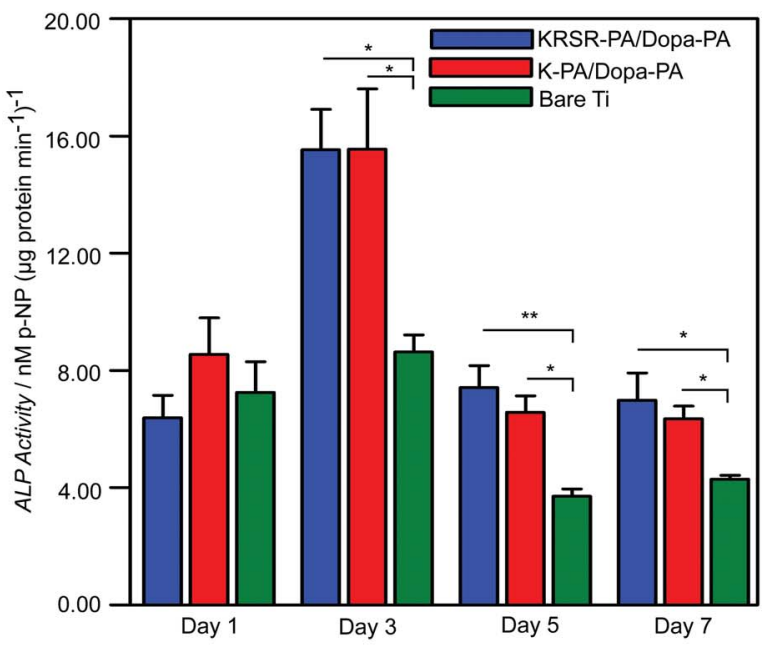

B)
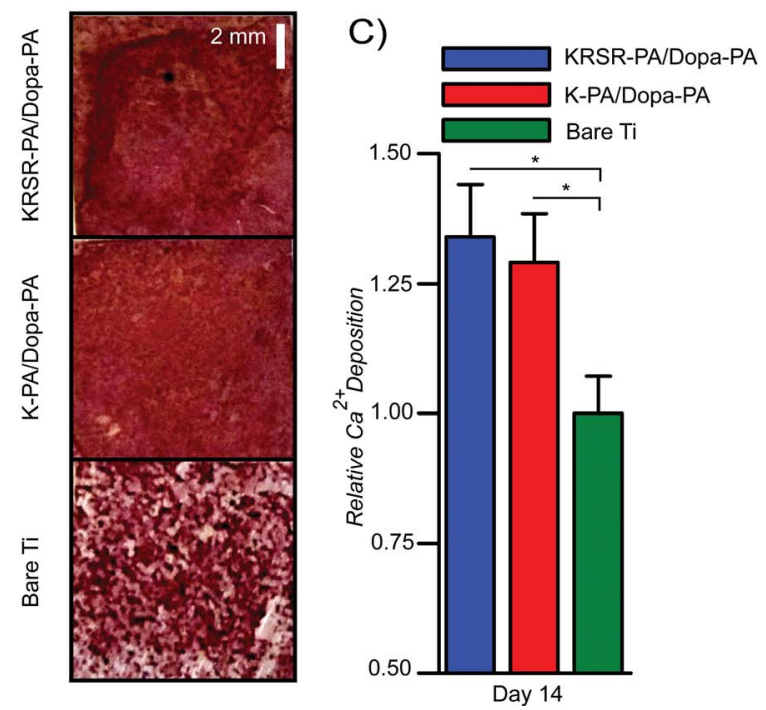

Fig. 5 Effect of immobilized PA nanofibers on osteogenic activity. (A) ALP activity of Saos2 cells on days 1, 3, 5 and 7. (B) Deposition of calcium on peptide coated titanium substrates on day 14 as demonstrated by Alizarin Red staining. (C) Quantification of relative calcium deposition on the matrix on day $14 ;{ }^{*} p<0.05,{ }^{* *} p<0.01$.

uncoated titanium surface. The bottom-up surface engineering strategy presented in this work consisted of gathering ECMderived osteoblast-specific peptide (KRSR) and mussel-inspired adhesive residue (Dopa) into ECM-mimetic peptide nanofibers under physiological conditions. This hybrid material was securely and homogeneously immobilized onto the titanium surface while maintaining its bioactive properties. This strategy can be extended to other surface immobilization systems owing to the versatile adhesive properties of Dopa and the ease of ligand conjugation into peptide amphiphile molecules. By modifying the bioactive region of the peptide nanofiber system, a wide range of bioactive nanomaterials can be immobilized on various biomedical implants and devices. Therefore, our strategy offers a general route for biofunctionalization of biomedical material surfaces using bottom-up fabricated self-assembled peptide nanofibers that can be functionalized in accordance with the application of interest. 


\section{Experimental section}

\section{Materials}

All protected amino acids, lauric acid, [4- $\left[\alpha-\left(2^{\prime}, 4^{\prime}\right.\right.$-dimethoxyphenyl) Fmoc-aminomethylphenoxyacetomidonorleucyl-MBHA resin (Rink amide MBHA resin), 2-(1H-benzotriazol-1-yl)1,1,3,3-tetramethyluroniumhexafluorophosphate (HBTU) and diisopropylethylamine (DIEA) were purchased from NovaBiochem, ABCR, or Sigma-Aldrich. Medical grade TiAl6V4 alloy was purchased from Goodfellow. All other chemicals and materials used in this study were analytical grade and purchased from Invitrogen, Fisher, Merck, Alfa Aesar, and/or SigmaAldrich.

\section{Synthesis and characterization of peptide-amphiphile building blocks}

Peptide amphiphile molecules were manually synthesized using a standard Fmoc-protected solid phase peptide synthesis method. Amino acid couplings were performed with 2 equivalents of amino acids activated with 1.95 equivalents of HBTU, and 3 equivalents of DIEA for 1 equivalent of starting resin. The coupling time for each amino acid was $2 \mathrm{~h}$. Lauric acid addition was performed similarly to amino acid coupling except that the coupling time was $4 \mathrm{~h}$. Fmoc removal was performed with $20 \%$ piperidine-dimethylformamide (DMF) solution for $20 \mathrm{~min} .10 \%$ acetic anhydride-DMF solution was used to permanently acetylate the unreacted amine groups after each coupling step. DMF and dichloromethane (DCM) were used as washing solvents. Cleavage of protecting groups and peptide molecules from the resin was carried out by $95 \%$ trifluoroacetic acid-containing cleavage cocktail $(95 \%$ TFA, 2.5\% water, 2.5\% triisopropylsilane) for $3 \mathrm{~h}$. Excess TFA removal was carried out by rotary evaporation. PAs in the remaining solution were precipitated in ice-cold diethyl ether overnight. The precipitate was collected the next day by centrifugation and dissolved in ultrapure water. This solution was frozen at $-80{ }^{\circ} \mathrm{C}$ for $4 \mathrm{~h}$ and then lyophilized for one week. Synthesized batches were characterized by using a quadruple time of flight (Q-TOF) mass spectrometer with electrospray ionization (ESI) source equipped with a reversephase analytical high performance liquid chromatograph (HPLC). In order to remove residual TFA, positively-charged peptide amphiphiles were treated with $0.1 \mathrm{M} \mathrm{HCl}$ solution and lyophilized, negatively-charged PAs were purified with a preparative HPLC system (Agilent 1200 series). All peptide batches were freeze-dried and reconstituted in ultrapure water at $\mathrm{pH} 7.4$ before use.

\section{Formation of peptide nanofibers and their characterizations}

KRSR-PA/Dopa-PA nanofibers were formed by mixing KRSR$\mathrm{PA}$ and Dopa-PA at $1: 3$ ratios, respectively, which stabilizes all net charges at $\mathrm{pH}$ 7.4. For the same reason, KRSR-PA and E-PA were mixed at $2: 3$ ratios, respectively, to form KRSR-PA/E-PA, and K-PA and Dopa-PA were mixed at $1: 1$ ratios to form K-PA/Dopa-PA nanofibers. The samples for circular dichroism (Jasco J-815) were prepared by mixing KRSR-PA and Dopa-PA at $1 \times 10^{-5}$ and $3 \times 10^{-5} \mathrm{M}$ concentrations, respectively. Zeta potential measurements (Malvern Zeta-ZS) of individual PA solutions or their mixtures were performed at the given ratios above at concentrations in the order of $10^{-4} \mathrm{M}$. Frequency sweep rheology measurements (Anton Paar Physica RM301) were performed using PA mixtures at $10^{-3} \mathrm{M}$ concentration. SEM samples were prepared by mixing KRSR-PA/Dopa-PA at $1 \mathrm{mM}$ and $3 \mathrm{mM}$ concentrations, respectively, and then by critical-point drying following ethanol exchange. The samples were coated with 4-5 nm Au-Pd before imaging. Scanning transmission electron microscopy (STEM) images at HAADF mode were acquired with FEI Tecnai G2 F30 TEM at $300 \mathrm{kV}$. This mode enables better contrast in comparison with the conventional TEM and hence better structural analysis can be done. Samples for STEM were prepared by mixing $1 \mathrm{mM}$ KRSR-PA and DopaPA at $1: 3$ ratio, respectively, on a 200-mesh carbon TEM grid for $1 \mathrm{~min}$ followed by $2 \mathrm{wt} \%$ uranyl acetate staining for $30 \mathrm{~s}$ and drying immediately under nitrogen gas.

\section{Surface binding and characterization of peptide amphiphile- titanium substrates}

Medical grade TiAl6V4 titanium alloy (Goodfellow, UK) was used as titanium substrate. The substrate was truncated into $1 \mathrm{~cm}^{2}$ pieces and used after polishing. The substrates were successively cleaned using acetone, ethanol and water wash coupled with ultrasound sonication for $1 \mathrm{~h}$ each and then dried in a high vacuum oven at $100^{\circ} \mathrm{C}$ and $90 \mathrm{mbar}$ for 6-7 h. The surface binding tests of nanofibers onto surfaces were carried out against water competition. $1 \mathrm{mM}$ KRSR-PA and Dopa-PA solutions were mixed on a cleaned titanium surface at $1: 3$ ratios, respectively. The control of Dopa was designed using KRSR-PA and E-PA nanofibers, which were mixed at $1: 1.5$ ratios, respectively. The peptide nanofiber-titanium samples were kept in a humidified environment for $24-48 \mathrm{~h}$ in a Petri dish and were not allowed to dry. Then, the substrates were rinsed in water for $30 \mathrm{~min}$ and dried at $37{ }^{\circ} \mathrm{C}$ for a further $24 \mathrm{~h}$. The physical properties of the peptide nanofiber-modified surface that are formed against water competition (without drying and washing after incubation in a humid Petri dish) were characterized using X-ray photoelectron spectroscopy (XPS) (Thermo Scientific), scanning electron microscopy (SEM), attenuated total internal reflectance Fourier transform infrared spectroscopy (ATR-FTIR) (VORTEX 70), contact angle measurements (OCA 30 Dataphysics), and optical profilometry (Zygo New view 7200). Samples for optical profilometer were coated with $5 \mathrm{~nm} \mathrm{Au}-\mathrm{Pd}$ before measurement. SEM samples were prepared by an ethanol gradient and critical point drying (Tourismis Autosamdri ${ }^{\circledR}$ 815B) followed by 4-5 nm Au-Pd coating.

\section{Cell culturing and maintenance}

Saos2 human osteosarcoma cells (ATCC ${ }^{\circledR}$ HTB-85 ${ }^{\mathrm{TM}}$ ), MC3T3E1 mouse preosteoblastic cells and primary human gingival fibroblast cells (HGF) were used in adhesion, spreading, viability and proliferation experiments on PA coated titanium surface. HGF cells were isolated and characterized as described and were kindly provided as a gift from Prof. Dr A. U. Ural of GATA, Ankara, Turkey. ${ }^{41}$ All cells were cultured and propagated in $75 \mathrm{~cm}^{2}$ cell culture flasks using Dulbecco's Modified Eagle Serum (DMEM) supplemented with 10\% Fetal Bovine Serum (FBS), 
$1 \%$ penicillin/streptomycin and $2 \mathrm{mM}$ L-glutamine. The cells were grown at $37^{\circ} \mathrm{C}$ in a humidified chamber supplied with $5 \% \mathrm{CO}_{2}$. All cell experiments were carried out after $80-90 \%$ confluency was reached and cells were diluted $1: 3$ and $1: 4$ for subculturing.

\section{In vitro cell culture tests}

All in vitro tests described in this study including with Saos2, MC3T3-E1 and HGF cells were carried out on KRSR-PA/ Dopa-PA and K-PA/Dopa-PA coated and bare titanium surfaces. PA nanofibers were formed on cleaned titanium surface as described above and coated surfaces were allowed to dry in a chemical hood overnight. Further drying was done for $24 \mathrm{~h}$ at $37^{\circ} \mathrm{C}$. The mixing ratios of PAs were as described above. Before the experiments, the coatings were washed with PBS prior to the experiments to remove unbound nanofibers, which otherwise could interfere with cellular behavior as a soluble factor. Adhesion and spreading tests were performed under serum-free conditions for $1 \mathrm{~h}$. Before seeding cells for adhesion and spreading tests, they were incubated with serum-free DMEM medium, supplemented with $4 \mathrm{mg} \mathrm{mL}^{-1} \mathrm{BSA}$ and $50 \mu \mathrm{g} \mathrm{mL}^{-1}$ cyclohexamide for $1 \mathrm{~h}$ at standard cell culture conditions. Cyclohexamide, which is a well-known translation inhibitor, was used to limit the interference of endogenous proteins in adhesion and spreading of cells. BSA served to non-specifically block cell adhesion receptors. After $1 \mathrm{~h}$, cells were removed from tissue culture plate with trypsin/EDTA chemistry. Trypsin/EDTA was then removed by centrifugation and resuspension of cells in serum-free DMEM. The cells were then seeded on modified or unmodified titanium substrates (at density of $1.5 \times 10^{4}$ cells $\mathrm{cm}^{-2}$ ) located in 24-well plates in a serum-free DMEM medium. After $1 \mathrm{~h}$, the substrates were washed with PBS, and then were stained using Calcein AM for visualization. The viability experiments were carried out at $24 \mathrm{~h}$ in 10\% FBS/DMEM. Cells seeded on substrates at a density of $1.0 \times 10^{4} \mathrm{cells} \mathrm{cm}^{-2}$ were washed with PBS after $24 \mathrm{~h}$ followed by Calcein AM staining. Relative cell adhesions and viability were quantified by directly counting the number of cells on different locations (at least five or six random locations were photographed per well, and at least six wells were used per independent experiment) as described previously. ${ }^{17}$ The counts were then normalized to the bare titanium surface results. For probing the spreading and cellular morphology, cells were either fixed with 3.7\% formaldehyde followed by $10 \mathrm{~min}$ Triton X-100 permeabilization and TRITCconjugated phalloidin treatment (for confocal microscopy) or $2 \%$ gluteraldehyde/PBS followed by post fixation with osmium tetroxide (for SEM imaging).

ALP activity of Saos 2 cells was probed on day 1, 3, 5 and 7 by measuring the colorimetric product of $p$-nitrophenyl phosphate/ endogenous ALP reaction. The ALP results were normalized to the total protein amount which was determined by BCA protein assay kit (Pierce) according to manufacturer's instructions. In brief, cells were lysed with M-PER Protein Extraction Kit (Pierce) containing 5\% protease inhibitor for $20-25 \mathrm{~min}$ on shaker after discarding the culture medium and washing with PBS. Then, collected lysates were centrifuged for $15 \mathrm{~min}$ at $14000 \mathrm{~g}$ to discard the cellular debris. Protein containing supernatant was taken and BCA protein assay was performed. $50 \mu \mathrm{l}$ protein samples and $150 p$-NP substrate were used together with $p$-NP standards to determine final ALP concentration after 30 min incubation.

Calcium deposition on the surface was measured on day 14 and day 21 using Alizarin Red staining as previously reported. ${ }^{42}$ Briefly, cells were fixed with ice-cold ethanol for $1 \mathrm{~h}$ and stained with $40 \mathrm{mM}$ Alizarin-Red S for $15 \mathrm{~min}$. After washing 4-5 times with double distilled water to get rid of non-specific Alizarin-Red binding, Alizarin Red bound Ca extraction was performed by using $10 \%(\mathrm{w} / \mathrm{v})$ cetylpyridinium chloride in $10 \mathrm{mM}$ sodium phosphate $(\mathrm{pH} \mathrm{7)}$ for $20 \mathrm{~min}$ at room temperature and the concentration of Alizarin-Red S was determined by measuring the absorbance at $562 \mathrm{~nm}$. For ALP and Alizarin Red staining assays, Saos 2 cells were seeded on titanium disks located in 24 well plates at a density of $1.5 \times 10^{4}$ cells cm$^{-2}$ in $10 \% \mathrm{FBS} /$ DMEM. Cells were kept growing in 10\% FBS/DMEM until they reached $100 \%$ confluency, after which the medium was replaced with fresh osteogenic medium containing $10 \mathrm{mM} \beta$-glycerophosphate, $0.2 \mathrm{mM}$ ascorbic acid and $100 \mathrm{nM}$ dexamethasone in $10 \% \mathrm{FBS} / \mathrm{DMEM}$. This medium was replenished every 3-4 days in the course of experiments.

\section{Statistical analyses}

Unless otherwise indicated, all quantitative values were presented as mean \pm s.e.m (standard error of means). All in vitro experiments were quantified with at least four replicates. All surface characterizations were performed on at least three different locations for each group of surface. Statistical analyses were performed using either one-way analysis of variance (ANOVA) or Student's t-test, wherever necessary. A $p$-value of less than 0.05 was considered statistically significant.

\section{Acknowledgements}

We would like to express our gratitude to Z. Erdogan for her help in LC-MS, M. Guler for help in TEM and STEM, and Dr B. Ortac for sectioning the titanium sheets. This project was supported by the Scientific and Technological Research Council of Turkey (TUBITAK) grant number 110M353 and COMSTECHTWAS grant. H. C. and S. K. are supported by TUBITAKBIDEB fellowships. M. O. G. acknowledges support from the Turkish Academy of Sciences Distinguished Young Scientist Award (TUBA-GEBIP).

\section{References}

1 P. G. Laing, A. B. Ferguson Jr. and E. S. Hodge, J. Biomed. Mater. Res., 1967, 1, 135-149.

2 K. Hayashi, K. Uenoyama, N. Matsuguchi and Y. Sugioka, J. Biomed. Mater. Res., 1991, 25, 515-523.

3 E. D. Spoerke, N. G. Murray, H. Li, L. C. Brinson, D. C. Dunand and S. I. Stupp, Acta Biomater., 2005, 1, 523-533.

4 E. D. Spoerke and S. I. Stupp, Biomaterials, 2005, 26, 5120-5129.

$5 \mathrm{~K}$. Hayashi, T. Inadome, H. Tsumura, Y. Nakashima and Y. Sugioka, Biomaterials, 1994, 15, 1187-1191.

6 S. Lavenus, G. Louarn and P. Layrolle, Int. J. Biomater., 2010, 2010.

7 Y. Wu, J. P. Zitelli, K. S. TenHuisen, X. Yu and M. R. Libera, Biomaterials, 2011, 32, 951-960.

8 H. Schliephake, D. Scharnweber, M. Dard, A. Sewing, A. Aref and S. Roessler, J. Biomed. Mater. Res. B: Appl. Biomater., 2005, 73, 88-96.

9 M. Morra, Eur. Cell. Mater., 2006, 12, 1-15. 
10 T. D. Sargeant, M. O. Guler, S. M. Oppenheimer, A. Mata, R. L. Satcher, D. C. Dunand and S. I. Stupp, Biomaterials, 2008, 29, 161-171.

11 T. D. Sargeant, S. M. Oppenheimer, D. C. Dunand and S. I. Stupp, J. Tissue Eng. Regener. Med., 2008, 2, 455-462.

12 T. A. Petrie, J. E. Raynor, D. W. Dumbauld, T. T. Lee, S. Jagtap, K. L. Templeman, D. M. Collard and A. J. Garcia, Sci. Transl. Med., 2010, 2, 45ra60.

13 G. A. Silva, C. Czeisler, K. L. Niece, E. Beniash, D. A. Harrington, J. A. Kessler and S. I. Stupp, Science, 2004, 303, 1352-1355.

14 T. Dvir, B. P. Timko, D. S. Kohane and R. Langer, Nat. Nanotechnol., 2010, 6, 13-22.

15 J. D. Hartgerink, E. Beniash and S. I. Stupp, Science, 2001, 294, 1684 1688.

16 A. Mata, Y. Geng, K. J. Henrikson, C. Aparicio, S. R. Stock, R. L. Satcher and S. I. Stupp, Biomaterials, 2010, 31, 6004-6012.

17 H. Ceylan, A. B. Tekinay and M. O. Guler, Biomaterials, 2011, 32, 8797-8805.

18 T. Kraai, C. Brown, M. Neeff and K. Fisher, Int. J. Pediatr. Otorhi., 2011, 75, 749-753.

19 F. M. Vale, M. Castro, J. Monteiro, F. S. Couto, R. Pinto and J. M. G. T. Rico, Biomaterials, 1997, 18, 1133-1135.

20 T. Pap, A. Claus, S. Ohtsu, K. Hummel, P. Schwartz, S. Drynda, G. Pap, A. Machner, B. Stein, M. George, R. Gay, W. Neumann, S. Gay and W. Aicher, Arthritis Res., 2003, 5, R163-R173.

21 R. A. Mostardi, S. O. Meerbaum, M. W. Kovacik and I. A. Gradisar, J. Biomed. Mater. Res., 1999, 47, 60-64.

22 K. C. Dee, T. T. Andersen and R. Bizios, J. Biomed. Mater. Res., 1998, 40, 371-377.

23 H. Shin, S. Jo and A. G. Mikos, Biomaterials, 2003, 24, 4353-4364.

24 G. Balasundaram and T. J. Webster, J. Biomed. Mater. Res., Part A, 2007, 80A, 602-611.

25 H. Lee, J. Rho and P. B. Messersmith, Adv. Mater., 2009, 21, 431-434.

26 S. M. Martin, J. L. Schwartz, C. M. Giachelli and B. D. Ratner, J. Biomed. Mater. Res., 2004, 70A, 10-19.
27 S. J. Xiao, M. Textor, N. D. Spencer, M. Wieland, B. Keller and H. Sigrist, J. Mater. Sci.: Mater. Med., 1997, 8, 867-872.

28 H. Lee, S. M. Dellatore, W. M. Miller and P. B. Messersmith, Science, 2007, 318, 426-430.

29 B. H. Choi, Y. S. Choi, D. G. Kang, B. J. Kim, Y. H. Song and H. J. Cha, Biomaterials, 2010, 31, 8980-8988.

30 J. Ryu, S. H. Ku, H. Lee and C. B. Park, Adv. Funct. Mater., 2010, 20, 2132-2139.

31 H. Lee, N. F. Scherer and P. B. Messersmith, Proc. Natl. Acad. Sci. U. S. A., 2006, 103, 12999-13003.

32 L. M. Hamming, X. W. Fan, P. B. Messersmith and L. C. Brinson, Compos. Sci. Technol., 2008, 68, 2042-2048.

33 G. N. Belibasakis, A. Johansson, Y. Wang, C. Chen, T. Lagergard, S. Kalfas and U. H. Lerner, Cytokine, 2005, 30, 56-63.

34 K. L. Niece, J. D. Hartgerink, J. J. J. M. Donners and S. I. Stupp, J. Am. Chem. Soc., 2003, 125, 7146-7147.

35 C. Fant, J. Hedlund, F. Höök, M. Berglin, E. Fridell and H. Elwing, J. Adhes., 2010, 86, 25-38.

36 A. B. Novaes Jr, S. L. S. d. Souza, R. R. M. d. Barros, K. K. Y. Pereira, G. Iezzi and A. Piattelli, Braz. Dent. J., 2010, 21, 471-481.

37 R. L. Price, M. C. Waid, K. M. Haberstroh and T. J. Webster, Biomaterials, 2003, 24, 1877-1887.

38 K. L. Elias, R. L. Price and T. J. Webster, Biomaterials, 2002, 23, 3279-3287.

39 T. P. Kunzler, T. Drobek, M. Schuler and N. D. Spencer, Biomaterials, 2007, 28, 2175-2182.

40 L. D. Quarles, D. A. Yohay, L. W. Lever, R. Caton and R. J. Wenstrup, J. Bone Miner. Res., 2009, 7, 683-692.

41 E. Zeldich, R. Koren, C. Nemcovsky and M. Weinreb, J. Dent. Res., 2007, 86, 41-46.

42 C. M. Stanford, P. A. Jacobson, E. D. Eanes, L. A. Lembke and R. J. Midura, J. Biol. Chem., 1995, 270, 9420-9428.

43 T. H. Anderson, J. Yu, A. Estrada, M. U. Hammer, J. H. Waite and J. N. Israelachvili, Adv. Funct. Mater., 2010, 20, 4196-4205. 